\title{
LEVANTAMENTO ENTOMOLÓGICO DOS VETORES DA DOENÇA DE CHAGAS DIAGNOSTICADOS NO MUNICÍPIO DE MOSSORÓ, ESTADO DO RIO GRANDE DO NORTE
}

\author{
ENTOMOLOGICAL SURVEY OF DIAGNOSED CHAGAS DISEASE \\ VECTORS IN MOSORÓ, RIO GRANDE DO NORTE STATE
}

Ismael Vinícius de Oliveira ${ }^{1}$

Ana Carla Diógenes Suassuna Bezerra ${ }^{2}$

\begin{abstract}
RESUMO: OBJETIVO: O objetivo da pesquisa foi descrever um levantamento entomológico de vetores da Doença de Chagas diagnósticados no município de Mossoró, Estado do Rio Grande do Norte. MÉTODO: O levantamento dos vetores foi realizado durante o período de cinco anos. Com a coleta realizada pelos moradores em distintos bairros, com relatos no domicílio e peridomicílio realizado em busca ativa eventual. Todos transportados vivos ao laboratório da universidade para diagnóstico do vetor e pesquisa de positividade para presença do protozoário Trypanosoma cruzi. RESULTADO: Foram diagnosticados um total de 62 barbeiros, entretanto 42 foram identificados como predadores Cosmoclopius nigroannulatus e 20 (vinte) hematófagos sendo 50\% (10/20) Triatoma pseudomaculata, 25\% (5/20) Triatoma brasiliensis e 15\% (3/20) Rhodnius nasutus e 10\% (2/20) do gênero Triatoma porém sem possibilidade de identiifcação até espécie. Dos hematófagos $100 \%(20 / 20)$ estavam negativos para diagnóstico de T. cruzi. CONCLUSÃO: No levantameno entomológico verificou-se o diagnóstico de vetores da Doença de Chagas na região domiciliar e peridominiliar. O que torna a região área de risco para patologia na região.
\end{abstract}

Palavras chave: Patologia; Risco Biológico; Triatominae.

ABSTRACT: OBJECTIVE: The objective of this research was to describe an entomological survey of diagnosed Chagas Disease vectors in the municipality of Mossoró, State of Rio Grande do Norte. METHOD: The vector survey was carried out during a five-year period. With the collection made by the residents in different neighborhoods, with reports at home and peridomiciliary conducted in an eventual

Discente da Pós-Graduação em Ambiente, Tecnologia e Sociedade; E-mail: viniciusunp@hotmail.com

2 Docente da Universidade Federal Rural do Semi-Árido; E-mail: anacarla@ufersa.edu.br. 
active search. All were transported alive to the university laboratory for vector diagnosis and positivity research for the presence of the Trypanosoma cruzi protozoan. RESULT: A total 62 barbers were diagnosed, however 42 were identified as Cosmoclopius nigroannulatus predators and 20 (twenty) hematophagous being $50 \%$ (10/20) Triatoma pseudomaculata, 25\% (5/20) Triatoma brasiliensis, 15\% (3/20) Rhodnius nasutus and $10 \%$ (2/20) of the genus Triatoma but without the possibility of identification to species. Of the 100\% hematophagous (20/20) were negative for the diagnosis of T. cruzi. CONCLUSION: In the entomological survey it was verified the diagnosis of Chagas disease vectors in the domiciliary and peridominilar region. Which makes the region at risk for pathology in the region.

Keywords: Pathology; Biological risk; Triatominae. 


\section{INTRODUÇÃO}

Zoonose causada pelo protozoário Trypanosoma cruzi, Doença de Chagas pode ser considerada uma das mais importantes patologia descoberta na América (SANTANA et al., 2019). De abrangencia ampla principalmente nos países tropicais, desde o Chile até México, com diagnóstico de milhões de pessoas infectadas, principalmente na América Latina (BRICEÑO- LEÓN; GALVÁN, 2007). No Brasil, os casos crônicos da doença são predominantes, com um número aproximado de três milhões de indivíduos acometidos e aumento dos casos agudos da patologia causada pela ingestão de alimentos contaminados (BRASIL, 2019).

Doença considerada negligenciada (SANTANA et al., 2019), porém com busca ao desenvolvimento científico para reduzir a enfermidade através de uma epidemiologia investigativa para maior compreensão dos principais locais e hospedeiros acometidos, drogas terapêuticas utilizadas, além de mapeamento de mutações e tropismo das cepas (BRASIL, 2019). Alterações das condições ambientais, incluindo as atividades antrópicas, podem ocasionar mudança nas comunidades biológicas dos insetos com adaptação de vetores silvestre em áreas peri e domiciliares (BERRY et al., 2019). Assim, a construção de residências em áreas rurais e o desmatamento são apontados como principais responsáveis da migração e adaptação dos insetos hematófagos as residências (GALVÃO, 2014).

Como consequência distintas linhagens de $T$. cruzi podem se estabelecer em áreas urbanizadas com expansão do vetor para regiões que antes não eram identificadas como áreas de risco (HODO et al., 2018). Nesse contexto, o diagnóstico de patologias causadas por vetores como a Doença de Chagas aumentou em áreas diversas, estabelecendo a importância de entender como os processos migratórios vetoriais afetam a epidemiologia das doenças nos ambientes urbanos (BERRY et al., 2019).

Os vetores no local de diagnóstico, presença de hospedeiros, condições sociais, econômicas e ambientais, associado a educação e saúde da população 
podem ser considerados fatores fundamentais para o risco de infecção chagásica (PÉREZ-MOLINA; MOLINA, 2018). Assim, para o controle existem alternativas eficazes como a pesquisa e combate vetorial, associado ao diagnóstico dos hospedeiros com testes precisos como os moleculares que podem ser empregados nos estudos epidemiológicos de transmissão e diagnóstico precoce, uma vez que, não há medidas preventivas eficientes como vacina (SCHIJMAN, 2018).

Com as vacinas ineficientes e a eficácia dos medicamentos disponibilizados limitada a fase aguda da doença, ocorre o estímulo ao desenvolvimento de pesquisas inovadoras como o bloqueio a transmissão em áreas endêmicas com molécula recombinante capaz de aderir à superfície do $T$. cruzi com estudos em andamentos (DEMEU et al., 2019). Assim, aumenta a importância das pesquisas dos insetos hematófagos com auxílio ao estudo epidemiológico vetorial em diversas regiões (BRASIL, 2019).

De acordo com Dias e colaboradores (2000), em termos de importância para saúde pública, as principais espécies vetoriais no Nordeste do Brasil são Triatoma brasiliensis (Neiva, 1911), Panstrongylus megistus (Burmeister, 1835), Triatoma infestans (Klug, 1834), Triatoma pseudomaculata (Corrêa; Espínola, 1964), Rhodnius nasutus (Stal, 1859) e Triatoma sordida (Stal, 1859).

No Estado do Rio Grande do Norte, os principais vetores capturados foram $T$. brasiliense, T. pseudomaculata, Panstrongylus lutzi, P. megistus e $R$. nasutus (FONSECA et al., 2010). Reforçando a importância do controle desses insetos de formacontínua nas áreas consideradas de risco, associado ao desenvolvimento de políticas de vigilância para diminuição dos casos de transmissão (SILVEIRA; DIAS, 2011).

Diante do exposto e levando em consideração que são insuficientes os dados sobre levantamento vetorial no Estado do Rio Grande do Norte o objetivo da pesquisa foi descrever um levantamento entomológico de vetores da Doença de Chagas diagnósticados no município de Mossoró, Estado do Rio Grande do Norte. 


\section{METODOLOGIA}

\section{DESCRIÇÃO DA ÁREA}

O estudo foi conduzido no município de Mossoró, estado do Rio Grande do Norte, região Nordeste do Brasil, cujas coordenadas são $05^{\circ} 11^{\prime} 16,8^{\prime \prime}$ de latitude sul e $37^{\circ} 20^{\prime} 38,4^{\prime \prime}$ de longitude oeste, situando-se na mesorregião Oeste Potiguar e abrangendo uma área de $2.100 \mathrm{~km}^{2}$ (MASCARENHAS et al., 2005).

Apresenta uma altitude de 16 metros, com clima predominante semiárido, temperatura média de $27,4^{\circ} \mathrm{C}$, estação chuvosa concentrada entre o verão, com regime de chuvas irregulares.

\section{LEVANTAMENTO DOS BARBEIROS}

Foi realizado o levantamento dos vetores durante o período de cinco anos (2015 a 2019). Os exemplares foram coletados por moradores domiciliados no município de Mossoró e transportados vivos ao Laboratório de Biotecnologia Aplicada a Doenças Infecto-Parasitárias da Universidade Federal Rural do SemiÁrido (UFERSA). A coleta realizada pelos moradores ocorreu em distintos bairros, com relatos de captura no domicílio e peridomicílio, realizado em busca ativa eventual.

\section{IDENTIFICAÇÃO DOS ESPÉCIMES COLETADOS}

A identificação ocorreu de acordo com a classificação de Lent e Wigodzinski (1979). Para os demais insetos foram utilizadas chaves entomológicas selecionadas de acordo com as características morfológicas dos insetos (LEITE; SÁ, 2010). 


\section{ANÁLISE DA POSITIVIDADE DOS VETORES HEMATÓFAGOS}

A pesquisa $T$. cruzi foi realizada nos triatomíneos ingurgitados e identificados como hematófagos. Realizada por compressão do abdômen e exame a fresco. Associado a dissecação do barbeiro para verificação definitiva através da análise do tubo digestivo, com maceração do duodeno e adição de duas gotas de soro fisiológico com posterior visualização em microscópio óptico (MAGALLÓNGASTÉLUM et al., 1998).

\section{RESULTADOS}

Foram diagnosticados um total de 62 barbeiros, entretanto 42 foram identificados como predadores Cosmoclopius nigroannulatus e 20 (vinte) hematófagos (Tabela 1)

Tabela 1 - Identificação dos triatomíneos hematófagos coletados por residentes do município de mossoró, Estado do Rio Grande do Norte, coletados em busca ativa eventual.

\begin{tabular}{ccc}
\hline VETORES & $\mathbf{n}$ & $\%$ \\
\hline Triatoma pseudomaculata & 10 & 50 \\
Triatoma brasiliensis & 05 & 25 \\
Rhodnius nasutus & 03 & 15 \\
Ninfas de Triatoma & 02 & 10 \\
\hline Dos hematófagos $100 \%(20 / 20)$ estavam negativos para diagnóstico de T. cruzi.
\end{tabular}

\section{DISCUSSÃo}

Cosmoclopius nigroannulatus foi o inseto mais diagnosticado no referido levantamento, entretando tem característica predatorial não hematófaga. Esses insetos apresentam um comportamento alimentar de predadores, sua disperssão 
está diretamente relacionada a abundância de presas e sua presença pode gerar impactos favorávies nas populações com as quais interagem com grande relevância no controle biológico (ROCHA et al., 2002).

C. nigroannulatus representa irrelevante importância para transmissão da Doença de Chagas. Uma vez que, para infecção do protozoário Trypanosoma cruzi ocorrer de forma vetorial esse precisa alimentar-se de sangue (ZELEDÓN; RABINOVICH, 1981), ou ocorre transfusões oriundo de hospedeiro infectado, via transplacentária, acidentes laboratoriais, manipulação de animais/hospedeiros infectados e/ou através de transplantes de órgãos além da transmissão por via oral considerada uma das principais do Brasil (BRASII, 2018).

C. nigroannulatus foi o mais encontrado nas residências do referido estudo. Que pode ser explicado devido a característica do inseto em selecionar locais protegidos para oviposição se deslocam para o espaço peri ou domiciliar. Assim, em contrato com o homem pode se defender principalmente quando em contato com as roupas, porém sem risco de transmitir $T$. cruzi.

Dentre os insetos hemófagos Triatoma pseudomaculata foi diagnosticado como o mais encontrado pela população no espaço domiciliar. Segundo Argolo e colaboradores (2008) é uma espécie de boa adaptação em regiões quentes e semiáridas, o que favorece ser um vetor bastante encontrado no estado do Nordeste do Brasil, com predileção por residências onde são encontrados nas partes mais quentes como os telhados. T. pseudomaculata tem como habitat natural áreas rurais ou silvestres, com ênfase a ecótopo como: abrigo de animais, palmeiras e ninhos de aves (BARBOSA et al., 2016), além de cascas de árvores, como a jurema (Mimosa tenuiflora) (FREITAS et al., 2004).

As residências onde são diagnosticados tem como características: cenário de pobreza acentuada, elevado número de comunidades rurais, índices de habitações humanas de baixa qualidade que são ideias para o abrigo de triatomíneos (DIAS et al., 2000). T. pseudomaculata foi considerado o segundo vetor mais coletado no espaço intradomiciliar do semiárido brasileiro (COSTA et al., 2003; SARQUIS et al., 2006).

O segundo mais encontrado foi Triatoma brasiliensis. Considerado um vetor que pode ser atraído pelas luzes residenciais (NEVES et al., 2016). T. brasiliensis é 
um vetor geralmente capturado no ambiente silvestre (BARBOSA et al., 2016), encontrado no semiárido nordestino onde destaca-se como predominantes e apresentam potencial invasivo e com difícil controle (DIAS et al., 2000).

O mesmo vetor foi diagnosticado em vários estados do Nordeste, entretanto em Campina Grande e municípios circunvizinhos do estado da Paraíba, onde Lins e colaboradores (2014) diagnosticaram a prevelência de 26,9\% (18 de 650) T. brasiliensis. Tem como característica apresentar maior atividade no final do dia, e prefere temperaturas mais elevadas com período de maior infestação na época de chuva (TEIXEIRA, 2007). Os mesmo autores verificaram também a positividade quanto a presença do protozoário T. cruzi observando no T. brasiliensis 50\% (9 de 18) e T. pseudomaculata 2,1\% (12 de 566).

Rhodnius nasutus foi o terceiro a ser observado no levantamento. A incidência de $R$. nasutus pode ser explicada através da presença de carnaúbas e ninho de aves no local pesquisado considerado local de vida do vetor (TEIXEIRA, 2007), e foi descrito por Dias e colaboradores (2008) como principal habitat na região de Caatinga no estado do Ceará (DIAS et al., 2008).

Pesquisas realizadas com infecção natural diagnosticaram as espécies $T$. pseudomaculata $(1,8 \%)$ e $R$. nasutus $(3,0 \%)$ no estado do Nordeste no Brasil, com estudo da predileção alimentar associando os roedores e marsupiais (FORATTINI et al., 1980).

Considerado vetor de importância em saúde púbica (DIAS et al., 2000). Quanto a positividade todos foram negativos, o que pode ser explicado em razão da ausência de alimentação dos vetores capturados no momento do diagnóstico, o que leva a alta possibilidade de resultado falso negativo. Nesse contexto, salienta-se que a doença de Chagas ter se tornado endêmica no município aos migrantes das zonas rurais ou agricultores que chegaram à cidade para atividades diversa. Com essa migração houve maior número de hospedeiros infectados e a associação com vetores adaptados ao domicílio levou ao aumento do número de casos na região. Tornando a pesquisa vetorial uma atividade importante dentro do controle epidemiológico da doença. 


\section{CONCLUSÃO}

No levantameno entomológico verificou-se o diagnóstico de vetores da Doença de Chagas na região domiciliar e peridominiliar.

\section{REFERÊNCIAS BIBLIOGRÁFICAS}

ARAÚJO, D. R. et al. Estudo da área de preservação permanente do rio Mossoró no sítio urbano de Mossoró-RN por meio de técnicas de geoprocessamento. Revista Caatinga, v.25, n.2, p.177- 183, 2012. http://periodicos.ufersa.edu.br/index.php/sistema

ARGOLO, A. M.; FELIX, M.; PACHECO, R.; COSTA, J. Doença de Chagas e seus principais vetores no Brasil, 1ª. ed., Rio de Janeiro: Imperial Novo Milênio, v. 1, 67p., 2008. DOI: 10.13140/2.1.1578.9449.

BARBOSA et al. Characteristics of Triatomine infestation and natural Trypanosoma cruzi infection in the State of Rio Grande do Norte, Brazil. Revista da Sociedade Brasileira de Medicina Tropical, 49(1): 57-67, 2016. DOI: http://dx.doi.org/10.1590/0037-8682-0300-2015.

BERRY, A.S.F. et al. Immigration and establishment of Trypanosoma cruzi in Arequipa, Peru PLoS ONE, v.14, n.8, e0221678. DOI: https://doi.org/10.1371/journal.pone.0221678.

BRASIL, Ministério da Saúde. Protocolo Clínico e diretrizes Terapêuticas Doença de Chagas. Secretaria de Ciência, Tecnologia e Insumos Estratégicos. 1 ed. Atual. - Brasília: Ministerio da Saúde, 2018. http://conitec.gov.br/images/Protocolos/Relatorio_PCDT_Doenca_de_Chagas.pdf.

BRASIL. Ministério da Saúd:. Secretaria de Vigilância em Saúde. Boletim Epidemiológico 40. v.50. 15p. 2019. http://www.saude.gov.br/boletins-epidemiologicos.

BRICEÑO-LEÓN, R.; GALVÁN, J.M. The social determinants of Chagas disease and the transformations of Latin America. Mem Inst Oswaldo Cruz. v. 102 (Suppl. I).p. 109-112, 2007. DOI: http://dx.doi.org/10.1590/S0074-02762007005000095.

COSTA, J. et al. The epidemiologic importance of Triatoma brasiliensis as a Chagas disease vector in Brazil: a revision of domiciliary captures during 1993-1999. Memórias do Instituto Oswaldo Cruz, 98:443-449, 2003. DOI: http://dx.doi.org/10.1590/S0074-02762003000400002.

COURA, J. R. Present situation and new strategies for Chagas disease chemotherapy a proposal. Memórias do Instituto Oswaldo Cruz, v. 104, n. 4, p. 549-554, 2009. DOI: http://dx.doi.org/10.1590/S0074-02762009000400002.

DEMEU, L.M.K. et al. Engineering a single-chain antibody against Trypanosoma cruzi metacyclic trypomastigotes to block cell invasion. Plos One. v.16, p. 1-15, 2019. DOI: https://doi.org/10.1371/journal. pone.0223773.

DIAS, J. C. P. et al. Esboço geral e perspectivas da Doença de Chagas no Nordeste do Brasil. 
Cadernos de Saúde Pública, v. 16, n. 2, p. 13-34, 2000. DOI: http://dx.doi.org/10.1590/S0102$311 \times 2000000800003$.

DIAS, F. B. S. et al. Ecological aspects of Rhodnius nasutus Stål, 1859 (Hemiptera: Reduviidae: Triatominae) in palms of the Chapada do Araripe in Ceará, Brazil. Memórias do Instituto Oswaldo Cruz. v. 103, n. 8, p. 824-30, 2008. DOI: S0074-02762008000800014.

FREITAS, S.P.C.; FREITAS, A. L. C.; PRAZERES, S. M.; GONÇALVES, T. C. M. Influência de hábitos antrópicos na dispersão de Triatoma pseudomaculata Corrêa \& Espínola, 1964, através de Mimosa tenuiflora (Willdenow) (Mimosaceae) no Estado do Ceará, Brasil. Caderno de Saúde Pública, 20:333-336, 2004. DOI: http://dx.doi.org/10.1590/S0034-89102005000100004.

FONSECA, Z.A.A.S. et al. Estudo da fauna dos triatomíneos recebidos no laboratório de entomologia do centro de controle de zoonoses no Município de Mossoró/RN. PUBVET, v.4, N. $\begin{array}{lllll}3, & \text { Ed. } & \text { Art. } & \text { 708, }\end{array}$ http://www.pubvet.com.br/uploads/4e6fd11ef5d073e122aa56fa5fc784c5.pdf.

FORATTINI, O. P. Biogeografia, origem e distribuição da domiciliação de triatomíneos no Brasil. Revista de Saúde Pública, 14:256-299, 1980. http://dx.doi.org/10.1590/S003489101980000300002.

GALVÃO, C. Vetores da doença de chagas no Brasil [recurso eletrônico]. Curitiba: Sociedade Brasileira de Zoologia, 2014. 289 p. - (Série Zoologia: guias e manuais de identificação / Sociedade Brasileira de Zoologia). http://books.scielo.org/id/mw58j/pdf/galvao9788598203096.pdf.

HODO, C.L. et al. Trypanosoma cruzi transmission among captive nonhuman primates, wildlife, and vectors. EcoHealth. v.15, n. 2, p. 426-436, 2018. DOI: https://doi.org/10.1007/s10393-018$1318-5$.

LEITE, G. L. D.; SÁ, V. G. M. Apostila: Taxonomia, Nomenclatura e Identificação de Espécies. Minas Gerais: Universidade Federal de Minas Gerais, Montes Claros. 50p, 2010.

LENT, H.; WIGODZINSKY, P. Revision of the triatominae (Hemiptera: Reduviidae) and their significance as vectors of Chagas disease. Bulletin of the American Museum of Natural History, v.163, p:125-520, 1979. DOI: http://hdl.handle.net/2246/1282.

LINS, I. V. F.; MOURA, G. B.; MEDEIROS, J. S. Prevalência e controle de vetores da doença de chagas na Paraíba. Biofarm, v. 10, n. 4, p.1-6, 2014. http://revista.uepb.edu.br/index.php/biofarm/article/view/2623/1394.

MAGALLÓN-GASTÉLUM, E. et al. Distribución de los vectores de la enfermedad de Chagas Hemiptera: Reduviidae: Triatominae en el estado de Jalisco, México. Revista Biomédica, 9:151-157, 1998. DOI: http://www.uady.mx/ biomedic/rb98932.html.

MASCARENHAS, J. C. et al. (Org.) Projeto cadastro de fontes de abastecimento por água subterrânea. Diagnóstico do município de Mossoró, estado do Rio Grande do Norte. Recife: CPRM/PRODEEM, 2005. 11p. ilust.

NEVES, D. P.; MELO, A.L.; LENARDI, P.M.; VITOR, R.W.A. Parasitologia Humana. 13. ed. São Paulo: Atheneu, 2016. 616p.

PÉREZ-MOLINA, J. A.; MOLINA, I. Chagas disease. Lancet. v.391, p. 82-94, 2018. DOI: http://dx.doi.org/10.1016/ S0140-6736(17)31612-4.

ROCHA, L. et al. Extração de Alimento por Cosmoclopius nigroannulatus Stal (Hemiptera: Reduviidae) de Ninfas de Spartocera dentiventris (Berg) (Hemiptera: Coreidae). Neotropical Entomology. v. 31, n. 4. p.601-607, 2002. https://www.lume.ufrgs.br/handle/10183/86828. 
SARQUIS, O.; SPOSINA, R.; OLIVEIRA, T. G.; MAC CORD, J. R.; CABELLO, P. H.; BORGESPEREIRA, J.; et al. Aspects of peridomiciliary eco-topes in rural areas of Northeastern Brazil associated to triatomine (Hemiptera: Reduviidae) infestation, vectors of Chagas disease. Memórias do Instituto Oswaldo Cruz, 101:143-147, 2006. DOI: http://dx.doi.org/10.1590/S0074-02762006000200005.

SANTANA et al. Oral Transmission of Trypanosoma cruzi, Brazilian Amazon. Emerging Infectious Diseases. v. $25, \quad$ n. 1 , p. 132-135, $2019 . \quad$ DOI http://dx.doiorg.ez13.periodicos.capes.gov.br/10.3201/eid2501.180646.

SCHIJMAN, A. G. Molecular diagnosis of Trypanosoma cruzi. Acta Tropica. v 184, p. 59-66, 2018. DOI: https://doi.org/10.1016/j.actatropica.2018.02.019.

SILVEIRA A. C.; DIAS, J. C. P. O controle da transmissão vetorial. Revista da Sociedade Brasileira de Medicina Tropical, v. 44, p. 52-63, 2011. DOI: http://dx.doi.org/10.1590/S003786822011000800009 .

TEIXEIRA. A. R. L.; Doença de Chagas e evolução. Brasília: Universidade de Brasília, 309p, 2007.

ZELEDÓN, R.; RABINOVICH, J.E. Chagas diasease: an ecological appraisal with special emphasis os its insect vectors. Annual Review of Entomology, Palo Alto, v.26, p.101-133, 1981.

https://www.academia.edu/12567682/Chagas_Disease_an_Ecological_Appraisal_With_Special _Emphasis_on_its_Insect_Vectors. 